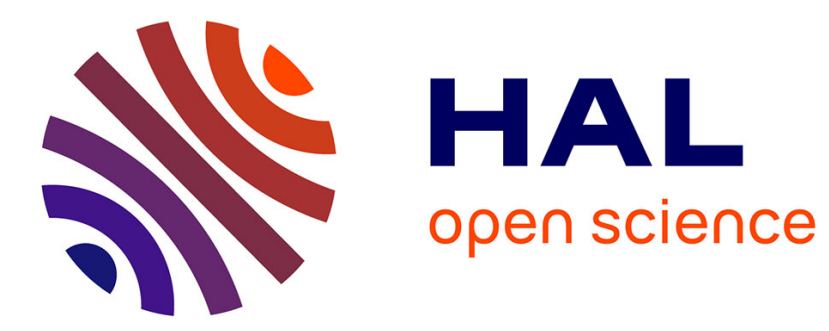

\title{
Approximate pricing formula to capture leverage effect and stochastic volatility of a financial asset
}

Youssef El-Khatib, Stéphane Goutte, Zororo S Makumbe, Josep Vives

\section{To cite this version:}

Youssef El-Khatib, Stéphane Goutte, Zororo S Makumbe, Josep Vives. Approximate pricing formula to capture leverage effect and stochastic volatility of a financial asset. 2021. halshs-03211698

\section{HAL Id: halshs-03211698 \\ https://shs.hal.science/halshs-03211698}

Preprint submitted on 29 Apr 2021

HAL is a multi-disciplinary open access archive for the deposit and dissemination of scientific research documents, whether they are published or not. The documents may come from teaching and research institutions in France or abroad, or from public or private research centers.
L'archive ouverte pluridisciplinaire HAL, est destinée au dépôt et à la diffusion de documents scientifiques de niveau recherche, publiés ou non, émanant des établissements d'enseignement et de recherche français ou étrangers, des laboratoires publics ou privés. 


\title{
Approximate pricing formula to capture leverage effect and stochastic volatility of a financial asset
}

\author{
Youssef El-Khatib ${ }^{1,2}$, Stephane Goutte ${ }^{3}$, Zororo S. Makumbe ${ }^{4,2}$, Josep \\ Vives $^{4}$
}

\begin{abstract}
In this paper a hybrid model is investigated to capture both financial behaviors of an asset: (i) the leverage effect and (ii) the stochastic volatility component. For this we consider a hybrid model that takes the strengths of the Heston and the CEV models. The pricing of European options is investigated both theoretically and empirically. A decomposition formula that allows to estimate the option price is obtained. Moreover, numerical simulations of the asset price are done to give a better and concrete vision of the adding of this approach. In addition, the price of a European call option under the hybrid model is computed using the Monte Carlo method and our formula. Illustrations and tables show the efficiency of the numerical method based on our approximate formula.
\end{abstract}

Keywords: Heston-CEV model, Stochastic volatility, European options, Monte Carlo method, Decomposition formula

\section{Introduction}

The Black-Scholes option pricing formula (see Black and Scholes (1973)) is extensively utilised by practitioners. Nevertheless, many of its assumptions have embedded some weaknesses into the model. For example it is assumed that returns are log normal but in practice they are not and rather tend to be leptokurtic and hence outliers are more common than expected. The

\footnotetext{
${ }^{1}$ Corresponding author, email: youssef_elkhatib@uaeu.ac.ae.

${ }^{2}$ United Arab Emirates University. Dep. of Mathematical Sciences, Al-Ain, UAE.

${ }^{3}$ Université Paris-Saclay, CEMOTEV, Paris, France.

${ }^{4}$ Universitst de Barcelona. Dep. de Matemàtiques i Informàtica, Barcelona, Spain.
} 
model also assumes that volatility is constant and was at first obtained by estimation using historical data and is known as realised volatility. However, empirical evidence suggests that this assumption is inconsistent with reality. Later implied volatility was used to estimate the volatility as it was a more futuristic estimate. However, plots of implied volatility against strike price were found to have a 'smile' which suggested that the assumption of constant volatility was not consistent with observed data. As a result of the above mentioned, there is a risk that the computed prices are not fair giving rise to the study of Stochastic Volatility. In this work, we focus our attention to the constant volatility problem. Several types of stochastic volatility models appeared to overcome this faulty assumption.

This paper considers a hybrid stochastic volatility model based on the Constant Elasticity of Variance (CEV) Cox (1975) and the Heston Heston (1993) models. It aims first at investigating the pricing of European options under the considered model. The second goal is to discuss the properties of the model (in other words the stylized facts satsified by the model) that address these limitations of the Black-Scholes model stated above. To study the options valuation, we provide an estimate of the option price using a decomposition method as in Alòs (2012), Merino and Vives (2015), Merino and Vives (2017). In addition, a numerical solution of the option price using Monte Carlo techniques is obtained. On the other hand, the study utilizes numerical methods for finance to provide simulations of the hybrid model and to explore several stylized facts satisfied by the model.

The rest of the paper is structured as follows: In section 2 we present the hybrid model as well as the properties of the hybrid model. In addition, in section (3) provides a decomposition formula for the hybrid HestonCEV stochastic model, which is used to estimate the option price. Section (4.1) presents numerical simulations for the Heston-CEV stochastic volatility model. In addition, we utilize the Monte Carlo method to price European options under the Heston-CEV stochastic volatility model. Several illustrations for asset price trajectories and the option price are provided. Lastly, section (5) concludes the paper. 


\section{The Hybrid Heston-CEV Model}

The Hybrid Heston-CEV model was studied in several papers among others Choi et al. (2013) and El-Khatib and Hatemi-J (2014), and in other related works like Lord et al. (2010). Choi et al. (2013) applied asymptotic methods to the pricing problem and their volatility is driven by the OrnsteinUhlenbeck process. On the other hand, El-Khatib and Hatemi-J (2014) considers finding the minimal equivalent martingale measure, the hedging, and derivation of the price PDE. Their volatility is driven by the CIR process unlike the Choi et al. (2013) case. Consider two independent Brownian motions $W:=\left(W_{t}\right)_{t \in[0, T]}$ and $B:=\left(B_{t}\right)_{t \in[0, T]}$ defined on the filtered probability space $\left(\Omega, \mathcal{F},\left(\mathcal{F}_{t}\right)_{t \in[0, T]}, P\right)$, and assume that $\left(\mathcal{F}_{t}\right)_{t \in[0, T]}$ is the natural filtration generated by $W$ and $B$. Moreover, we suppose that $\mathcal{F}=\mathcal{F}_{T}$. As in El-Khatib and Hatemi-J (2014), we consider the Hybrid Heston-CEV model as follows:

$$
\begin{aligned}
& d S_{t}=r S_{t} d t+\sqrt{\nu_{t}} S_{t}^{\alpha} d Z_{t} \\
& d \nu_{t}=\kappa\left(\theta-\nu_{t}\right) d t+\xi \sqrt{\nu_{t}} d W_{t} .
\end{aligned}
$$

Where $\kappa$ is the mean reversion rate, $\theta$ is long run variance, and $\xi$ is the volatility of variance. Like the SABR model the Weiner processes are correlated which is meant to take into account the leverage effect, that is

$\mathbb{E}\left[d Z_{t} d W_{t}\right]=\rho d t$ where $|\rho|<1$ and thus we set $Z_{t}=\rho W_{t}+\sqrt{1-\rho^{2}} B_{t}$. Next, we discuss some important financial properties of the hybrid model.

\subsection{Some Properties of the Model}

We can remark that taking parameter $\alpha=1$ gives us the classical Heston model. This model is calibrated to match market dynamics by taking into consideration the following characteristics where $\kappa$ regulates the skew and must be very small, while $\xi$ must be significant in value, and $\theta$ is very close to the implied volatility. These parameters are chosen such that $2 \kappa \theta>\xi$, which is known as the Feller condition. The Heston model achieves calibration to today's observed plain vanilla option prices by balancing the probabilities of very high volatility scenarios against those where future instantaneous volatility drops to very low levels. On the other hand, the CEV model was introduced by Cox (1975) to capture the leverage effect where underlying asset price is obtained from our SDE (1) where $\sqrt{\nu}$ is replaced by constant volatility $\sigma$. In this case, $\alpha$ is known as the elasticity parameter and $\sigma$ is the volatility scale parameter. Generally, in order to capture the leverage 
effect we require that $\alpha<1$. For different values of $\beta$ the $\mathrm{CEV}$ model reduces to other models covered in existing literature. When $\alpha=0$ the CEV model reduces to the constant volatility geometric Brownian motion process employed in the Black, Scholes and Merton model, when $\alpha=-1$, we have Bachelier's model (the asset price has the constant diffusion coefficient, while the logarithm of the asset price has the a/S volatility) and for $\alpha=-\frac{1}{2}$ we have the square-root model of Cox and Ross. Of the several well known stylized facts that we desire to verify, we consider the leverage effect and the volatility clustering property. In Figure 1a. we notice that the simulated returns are non-normal in that their distribution has a higher peak and is not perfectly symmetrical. In addition, the q-q plot of returns in Figure 1b. verify the non-normal distribution.

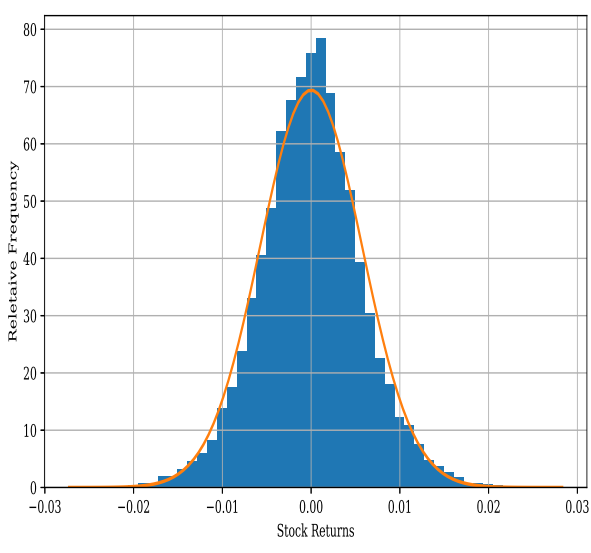

(a) Histogram of returns

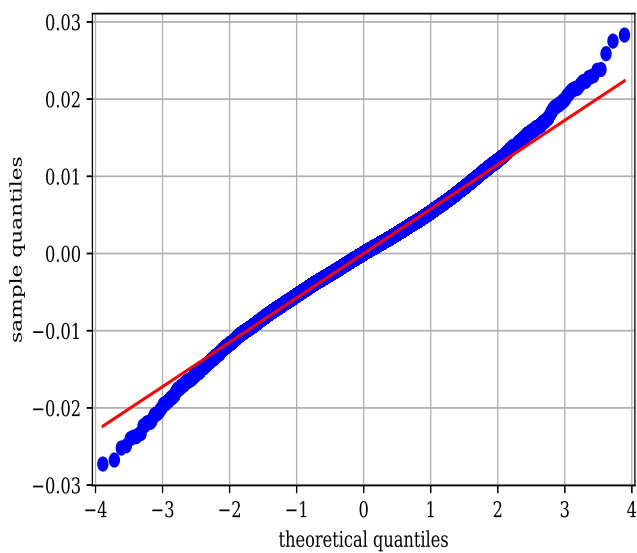

(b) Quantile-quantile plot of returns

Figure 1: $S_{0}=2.9, K=2.8$ and $\alpha=0.5$ when $\nu_{0}=0.16, r=0.06, \theta=0.16, \kappa=1, \xi=2$, $\rho=-0.8$

Next, we consider the leverage effect where we find that the hybrid model inherits leverage properties from the CEV and Heston models.

Proposition 1. The returns of the Hybrid Heston-CEV model (1)-(2) satisfy the leverage effect provided $\alpha<1$ and $\rho<0$.

Proof. We can take returns to be given as $\hat{R}_{t}=\ln \left(S_{t}\right)$, but, in order to study the volatility characteristics of the returns, it is sufficient to instead define $R_{t}=\ln \left(S_{t}\right)-\int_{0}^{t}\left(r-\frac{1}{2} \nu_{u} S_{u}^{2(\alpha-1)}\right) d u$. It is known that instantaneous 
volatility is defined as:

$$
\lim _{\Delta t \rightarrow 0} \sqrt{\frac{\left(R_{t+\Delta t}-R_{t}\right)^{2}}{\Delta t}}=\sqrt{\frac{d R_{t}^{2}}{d t}}
$$

Which in our case implies that the volatility of returns is $\sigma_{t}=\sqrt{\nu_{t}} S_{t}^{\alpha-1}$. By Itô formula the return dynamics are given by the following pair of stochastic differential equations:

$$
d R_{t}=\sqrt{\nu_{t}} e^{(\alpha-1) \hat{R}_{t}} d Z_{t}, \quad d \nu_{t}=\kappa\left(\theta-\nu_{t}\right) d t+\xi \sqrt{\nu_{t}} d W_{t} .
$$

Clearly, $R_{t}$ has zero expectation and hence

$$
\operatorname{Var}\left(\int_{0}^{t} R_{u} d u\right)=\mathbb{E}\left[\left(\int_{0}^{t} \sqrt{\nu_{u}} e^{(\alpha-1) \hat{R}_{u}} d Z_{u}\right)^{2}\right]=\mathbb{E}\left[\int_{0}^{t} \nu_{u} e^{2(\alpha-1) \hat{R}_{u}} d u\right] .
$$

Variance has an inverse relationship with stock price if $\alpha<1$ and $\rho<0$.

Figure 2 illustrates the leverage effect for the hybrid model.
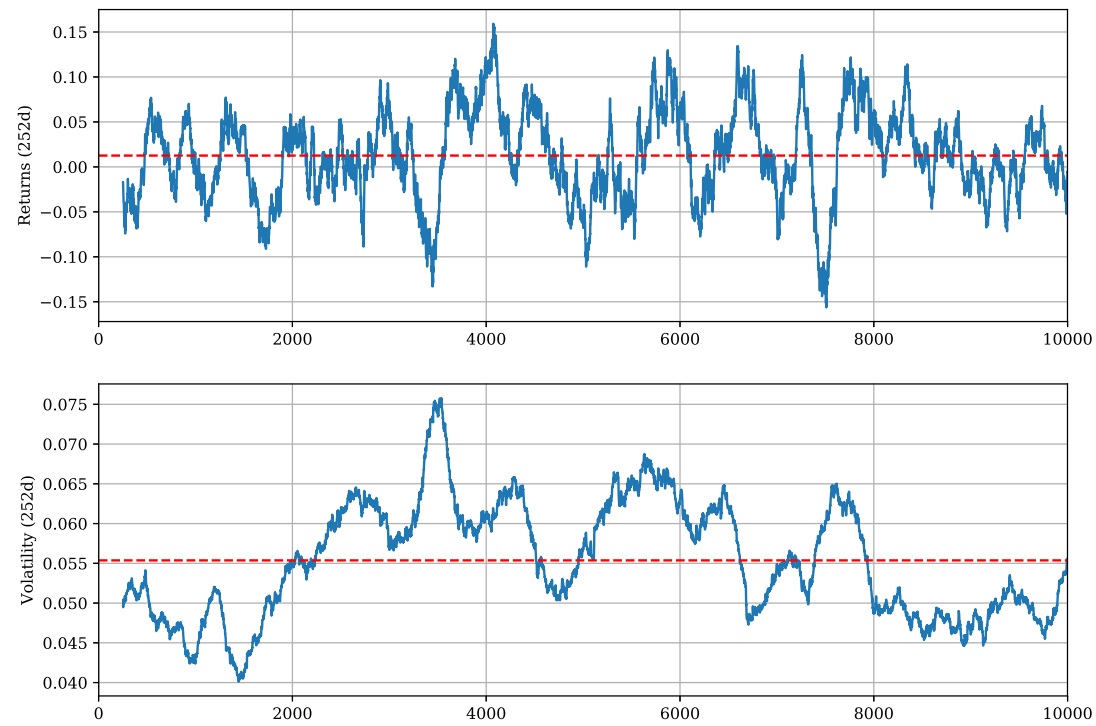

Figure 2: Rolling mean returns for $S_{0}=2.9, K=2.8$ and $\alpha=0.5$ when $\nu_{0}=0.16$, $r=0.06, \theta=0.16, \kappa=1, \xi=2, \rho=-0.8$ 
It also shows the volatility clustering characteristics. We notice that, large changes in the asset returns are followed by large changes and small changes are followed by small changes (there is a inverse relationship between returns and volatility).

\section{Decomposition formula for the option price}

In this section we deal with the derivation of a decomposition formula for European option price under our Hybrid Heston-CEV model. Let our model be defined as in (1)-(2). Merino and Vives (2015) obtained a general decomposition formula for similar models represented as

$$
d S_{t}=\mu\left(t, S_{t}\right) d t+\Theta\left(t, S_{t}, \nu_{t}\right) d Z_{t}
$$

However, they did not apply the decomposition. We will find an approximate formula and use it to price the option and then compare with the Monte Carlo approach. Our model is a special case where $\Theta\left(t, S_{t}, \nu_{t}\right)=\sqrt{\nu_{t}} S_{t}^{\alpha-1}$ and $\mu\left(t, S_{t}, \nu_{t}\right)=r S_{t}$. We may at times write $\Theta\left(t, S_{t}, \nu_{t}\right)=\Theta_{t}$ since the context is obvious. Let

$$
B S\left(t, S_{t}, \sigma\right)=S \Phi\left(d_{+}\right)-e^{-r t} \Phi\left(d_{-}\right)
$$

be the usual Black-Scholes formula with $\Phi(z)$ as the cumulative probability function of the standard normal distribution and

$$
d_{ \pm}=\frac{\ln (S / K)+\left(r \pm \sigma^{2} / 2\right)(T-t)}{\sigma \sqrt{T-t}} .
$$

Also, let

$$
\mathcal{L}_{\Theta}=\partial_{t}+\frac{1}{2} \Theta_{t}^{2} \partial_{S S}+r S \partial_{S}-r
$$

be the Feynman-Kac formula such that $\mathcal{L}_{\Theta} B S\left(t, S_{t}, \Theta_{t}\right)=0$. Define the following operators which will be useful in our presentation $\Gamma=S^{2} \partial_{S}^{2}, \Lambda=S \partial_{S}$ and $\Gamma^{2}=\Gamma \circ \Gamma$. We intend on pricing options driven by the Hybrid HestonCEV model as corrections of the Black-Scholes model following Merino and Vives (2017), Merino and Vives (2015), and Alòs (2012). So, we proceed as below. We know that the price of an European option where the stochastic volatility is independent of the asset price is given by

$$
P(t)=\mathbb{E}_{t}^{\mathbb{Q}}\left[B S\left(t, S_{t}, \bar{\nu}_{t}\right)\right]
$$


where $\bar{\nu}_{t}=\frac{1}{T-t} \int_{t}^{T} \nu_{s} d s$ is known as the future average variance and $\mathbb{E}_{t}[]=$. $\mathbb{E}\left[. \mid \mathcal{F}_{t}\right]$. Pricing in this case requires anticipative techniques so instead we define the adapted version of future average variance as $V_{t}=\frac{1}{T-t} \int_{t}^{T} \mathbb{E}\left[\bar{\nu}_{s} \mid \mathcal{F}_{t}\right] d s$ and change the problem into a non-anticipative one. So we approximate $\Theta\left(S_{t}, \nu_{t}\right)$ by $\Theta\left(S_{t}, V_{t}\right)$. Define also $M_{t}=\int_{0}^{T} \mathbb{E}\left[\nu_{s} \mid \mathcal{F}_{t}\right] d s$ such that $V_{t}=$ $\frac{1}{T-t}\left(M_{t}-\int_{0}^{T} \nu_{s} d s\right)$ and hence, $d V_{t}=\frac{1}{T-t}\left(d M_{t}+\left(V_{t}-\nu_{t}\right) d t\right)$. Now we are ready to present the decomposition formula in Merino and Vives (2015).

Theorem 1. For all $t \in(0, T]$, we have

$$
\begin{aligned}
\mathbb{E}_{t} & {\left[e^{-r T} B S\left(T, S_{T}, \nu_{T}\right)\right]=\frac{1}{2} \mathbb{E}_{t}\left[\int_{t}^{T} e^{-r u}\left(\frac{\Theta_{u}^{2}}{S^{2}}-\nu_{u}\right) \Gamma B S\left(u, S_{u}, \nu_{u}\right) d u\right] } \\
+ & \frac{\rho}{2} \mathbb{E}_{t}\left[\int_{t}^{T} e^{-r u} \frac{\Theta_{u}}{S_{u}} \Lambda \Gamma B S\left(u, S_{u}, \nu_{u}\right) d[M, W]_{u}\right] \\
+ & \mathbb{E}_{t}\left[\int_{t}^{T} \frac{e^{-r u}}{8} \Gamma^{2} B S\left(u, S_{u}, \nu_{u}\right) d[M, M]_{u}\right]+B S\left(t, S_{t}, \nu_{t}\right) .
\end{aligned}
$$

We, however, have a few simplifications widely applied in literature that make the formula more intuitive. For our own computational convenience we will obtain an approximate formula as follows:

Proposition 2 (Approximate Formula for the Hybrid model). The decomposition formula in Theorem (1) becomes

$$
\begin{aligned}
\mathbb{E}_{t}\left[e^{-r T} B S\left(T, S_{T}, \nu_{T}\right)\right] & =e^{-r t}\left(B S\left(t, S_{t}, \nu_{t}\right)+\left[S_{t}^{2(\alpha-1)}-1\right] \Gamma B S\left(t, S_{t}, \nu_{t}\right) C_{t}\right. \\
& \left.+S_{t}^{\alpha-1} \Lambda \Gamma B S\left(t, S_{t}, \nu_{t}\right) R_{t}+\Gamma^{2} B S\left(t, S_{t}, \nu_{t}\right) U_{t}\right)+\Omega
\end{aligned}
$$

where $\Omega$ is the error term and

$$
\begin{aligned}
C_{t} & =\frac{1}{2}\left(\theta(T-t)+\left(\nu_{t}-\theta\right) \varphi(t)\right) \\
R_{t} & =\frac{\rho \xi}{2 \kappa^{2}}\left(\theta \kappa(T-t)-2 \theta+\left(2 \theta-\nu_{t}\right) e^{-\kappa(T-t)}+\kappa(T-t)\left(\nu_{t}-\theta\right) e^{-\kappa(T-t)}\right) \\
U_{t} & =\frac{\xi^{3}}{8 \kappa^{2}}\left(\theta(T-t)+\left(\nu_{t}-3 \theta\right) \varphi(t)+\left(\nu_{t}-\theta\right)(\varphi(t)-2) e^{-\kappa(T-t)}+\theta \varphi_{2}(t)\right) \\
\varphi(t) & =\frac{1}{\kappa}\left(1-e^{-\kappa(T-t)}\right) \\
\varphi_{2}(t) & =\frac{1}{2 \kappa}\left(1-e^{-2 \kappa(T-t)}\right)
\end{aligned}
$$

Proof. Please refer to the appendix. 


\section{Simulation and Numerical results}

In this section we outline our approach to the Monte Carlo approximation of the option price. We first deal with the discretisation of the stochastic differential equations and simulation of the underlying asset price.

\subsection{Simulation of the hybrid Heston-CEV model}

Since the square-root process (2) is not globally Lipschitz then the convergence of the discretisation schemes is not guaranteed and it can cause the process to be negative which is undesirable. As a result, several fixes have been proposed namely absorption, reflection and full truncation as given by Lord et al. (2010). Broadie and Kaya (2006) proposed exact simulation method for the CIR process but Lord et al. (2010) and other researchers find that it is computationally intensive and the Euler-Maruyama technique yields good results especially with variance reduction methods. Andersen (2007) also propose a scheme based on the study of the properties of affine stochastic volatility models. Their approach is not of interest to us. We employ discretization schemes for (1) and (2) as follows:

- The naive Euler Maruyama:

$$
\begin{aligned}
& S_{i+1}=S_{i}+r S_{i} \Delta t+\sqrt{\nu_{i}^{+}} S_{i}^{\alpha} \Delta W_{1} \\
& \hat{\nu}_{i+1}=\hat{\nu}_{i}+\kappa\left(\theta-\hat{\nu}_{i}^{+}\right) \Delta t+b \sqrt{\hat{\nu}_{i}^{+}} \Delta W_{2}, \quad \nu_{i+1}=\hat{\nu}_{i+1}^{+} .
\end{aligned}
$$

- Kahl-Jackel Scheme using an Implicit Milstein scheme on the volatility and an additional "IJK" discretisation of the stock process.

$$
\begin{aligned}
& \ln S_{i+1}=\ln S_{i}+r S_{i} \Delta t+\sqrt{\nu_{i}^{+}} S_{i}^{\alpha} \Delta W_{1} \\
& \hat{\nu}_{i+1}=\frac{1}{1+\kappa \Delta t}\left[\hat{\nu}_{i}+\kappa\left(\theta-\hat{\nu}_{i}^{+}\right)\right] \Delta t+b \sqrt{\hat{\nu}_{i}^{+}} \Delta W_{2}, \quad \nu_{i+1}=\hat{\nu}_{i+1}^{+} .
\end{aligned}
$$

\subsection{Numerical Pricing of the European option}

The value of plain vanilla option price built on the hybrid model (1-2) cannot be calculated in closed form since the law of the random variable $S_{t}$ is not known. Hence, we utilise Monte Carlo and decomposition techniques to evaluate the option (3). In our work we will employ the Antithetic method as well as moment matching. Approximate the option price 
as $P \approx \frac{1}{2 N} \sum_{i=1}^{2 N} f\left(S_{i}(T)\right)$, where $2 N$ is the number of simulations carried out, and $S_{i}(T)=S\left(\omega_{i}, T\right)$ is the $i^{t h}$ a realisation of the terminal price of the asset obtained using the Euler-Maruyama discretization given in (??). In our simulations, $n=1000$ steps were one and the number of simulations was $2 N=2000$ with the terminal time being $T=1.0$. In order to reduce the number of necessary computations, variance reduction techniques on the generation of the uniform random variables were done. This has the added advantage of improving the convergence rate. Trajectories were generated as above for the approximation of the stock price path and realisations $S_{T}$ among other computations. We evaluate the option using the Monte Carlo Method as well as the decomposition technique discussed in section 3 using parameters obtained from Medvedev and Scaillet (2010). A Core i7 (8th Gen) CPU $1.90 \mathrm{GHz} 2.11 \mathrm{GHz}$ with 16GB RAM computer with Windows 10 (x64) is used to do the necessary computations in iPython and the results are obtained in table 1 which illustrates a comparison between two prices for the vanilla option of our Hybrid model (1-2) one using Monte Carlo methodology and the second price based on the approximate decomposition formula of Proposition. 2. We find that the Monte Carlo estimate and the decomposition estimate are very comparable for the most part differing in the third decimal place often. Applying the Milstein discretisation resulted in the exact same results.

\begin{tabular}{|c|c|c||c|c||c|c|}
\hline $\mathrm{T}$ & \multicolumn{2}{|c||}{$K=90$} & \multicolumn{2}{c||}{$K=100$} & \multicolumn{2}{c|}{$K=110$} \\
\hline \hline & $\mathrm{MC}$ & Decomp. & MC & Decomp. & MC & Decomp. \\
\hline 0.5 & 12.223 & 12.222 & 2.490 & 2.469 & 0.000 & 0.000 \\
\hline 1.0 & 14.374 & 14.389 & 4.877 & 4.877 & 0.000 & 0.000 \\
\hline 2.0 & 18.566 & 18.565 & 9.530 & 9.516 & 1.166 & 0.468 \\
\hline
\end{tabular}

Table 1: Call option prices when $S_{0}=100, \rho=-0.6, r=0.05, \kappa=1.5, \theta=0.02$, $\nu_{0}=0.04, \xi=0.15$

Analysing the difference in prices reveals that the decomposition provides a reasonable estimate under in the money (ITM) conditions and to a lesser extent during out of the money (OTM) conditions. However, the computational convenience is astounding. The decomposition calculation for all the values in Table 1 took 0.02 seconds while the same computations took 2.80 seconds via Monte Carlo. As a result, the decomposition method under this model is a viable alternative in short maturity pricing of ITM and at the 
money options. OTM option pricing error is higher for long maturities.

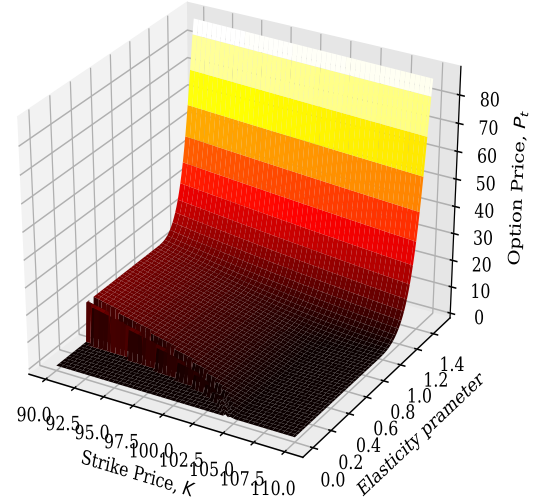

(a) Option price against $K \&$ Elasticity $\alpha$

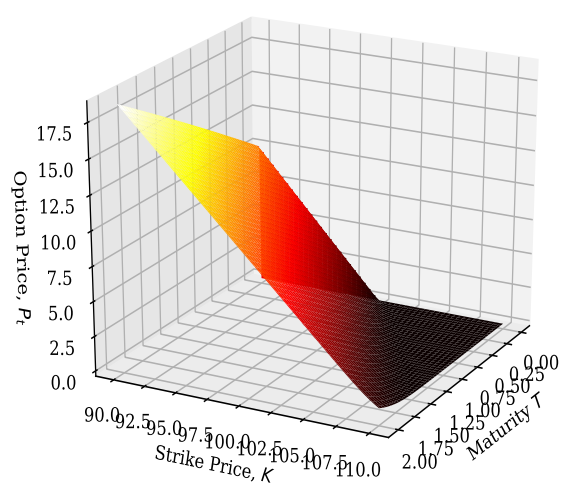

(b) Option price against $K \& T$

Figure 3: Plot of the option price against various parameters where for various $S_{0}, K$ when $\nu_{0}=0.16, r=0.06, \theta=0.16, \kappa=1, \xi=2, \rho=-0.8$

In addition, the decomposition enables plots of the price as shown in Figure $3 \mathrm{a}$ and $3 \mathrm{~b}$ which would take a great deal of time under Monte Carlo methods.

\section{Conclusions}

Stochastic volatility models are a strong tool that offers better representation of financial asset price fluctuations. However, solving pricing problem under such models is more complicated and in general closed form solutions are not available. In this paper we have studied a hybrid Heston-CEV stochastic volatility model. A decomposition formula is derived and is used in the estimation of option prices. Numerical simulations are conducted and show that the Heston-CEV covers more stylized facts compared to Black-Scholes or the Heston or CEV models alone. Moreover several illustrations comparing vanilla option prices by Monte Carlo method and by Proposition 2 demonstrates the efficiency of our approximate formula.

\section{Appendix: Proof of Proposition. 2 (approximate formula)}

Let $A\left(t, S_{t}, \nu_{t}\right)$ be function in $C^{(1,2,2)}$ and let $G_{t}$ be a semi-martingale. For the purposes of brevity we will instead write $A_{t}=A\left(t, S_{t}, \nu_{t}\right)$ and define a 
generic decomposition formula as

$$
\begin{aligned}
& \mathbb{E}_{t}\left[e^{-r T} A_{T} G_{T}\right]=e^{-r t} A\left(t, S_{t}, \nu_{t}\right) G_{t}+\mathbb{E}_{t}\left[\int_{t}^{T} e^{-r u} A_{u} d G_{u}\right] \\
& +\frac{1}{2} \mathbb{E}_{t}\left[\int_{t}^{T} e^{-r u}\left(\frac{\Theta_{u}^{2}}{S^{2}}-\nu_{u}\right) \Gamma A_{u} G_{u} d u\right]+\frac{1}{2} \mathbb{E}_{t}\left[\int_{t}^{T} e^{-r u} \Gamma A_{u} d[G, M]_{u}\right] \\
& +\frac{\rho}{2} \mathbb{E}_{t}\left[\int_{t}^{T} e^{-r u} \Theta_{u} \Lambda \Gamma A_{u} G_{u} d[M, W]_{u}\right]+\frac{1}{8} \mathbb{E}_{t}\left[\int_{t}^{T} e^{-r u} \Gamma^{2} A_{u} G_{u} d[M, M]_{u}\right] \\
& +\frac{1}{2} \mathbb{E}_{t}\left[\int_{t}^{T} e^{-r u} e^{-r u} \Theta_{u} \Lambda A_{u} d[G, Z]_{u}\right]
\end{aligned}
$$

For term I in Theorem 1, let $A_{t}=\left(S_{t}^{2(\alpha-1)}-1\right) \Gamma B S_{t}$, and $G_{t}=C_{t}=$ $\frac{1}{2} \mathbb{E}_{t}\left[\int_{t}^{T} \nu_{u} d u\right]$. Setting $\varphi(t)=\frac{1}{\kappa}\left(1-e^{-\kappa(T-t)}\right)$ it follows that,

$$
\begin{aligned}
C_{t} & =\frac{1}{2}\left(\theta(T-t)+\left(\nu_{t}-\theta\right) \varphi(t)\right) \\
d C_{t} & =-\frac{\nu_{t}}{2} d t+\frac{\xi}{2} \varphi(t) \sqrt{\nu_{t}} d W_{t}
\end{aligned}
$$

Thus we have

$$
\begin{aligned}
0 & =e^{-r t} C_{t} \Gamma B S_{t}-\frac{1}{2} \mathbb{E}_{t}\left[\int_{t}^{T} e^{-r u} \nu_{u}\left(\left(S_{u}^{2(\alpha-1)}-1\right) \Gamma B S_{u}\right) d u\right] \\
& +\frac{1}{4} \mathbb{E}_{t}\left[\int_{t}^{T} e^{-r u} C_{u}\left(\frac{\Theta_{u}^{2}}{S^{2}}-\nu_{u}\right) \Gamma\left(\left(S_{u}^{2(\alpha-1)}-1\right) \Gamma B S_{u}\right) d u\right] \\
& +\frac{\rho}{4} \mathbb{E}_{t}\left[\int_{t}^{T} e^{-r u} C_{u} \Theta_{u} \Lambda \Gamma\left(\left(S_{u}^{2(\alpha-1)}-1\right) \Gamma B S_{u}\right) d[M, W]_{u}\right] \\
& +\frac{1}{16} \mathbb{E}_{t}\left[\int_{t}^{T} e^{-r u} C_{u} \Gamma^{2}\left(\left(S_{u}^{2(\alpha-1)}-1\right) \Gamma B S_{u}\right) d[M, M]_{u}\right] \\
& +\frac{\xi}{4} \mathbb{E}_{t}\left[\int_{t}^{T} e^{-r u} \varphi(u) \sqrt{\nu_{u}} \Theta_{u} \Lambda\left(\left(S_{u}^{2(\alpha-1)}-1\right) \Gamma B S_{u}\right) d u\right] \\
& +\frac{\xi}{4} \mathbb{E}_{t}\left[\int_{t}^{T} e^{-r u} \varphi(u) \sqrt{\nu_{u}} \Gamma\left(\left(S_{u}^{2(\alpha-1)}-1\right) \Gamma B S_{u}\right) d[W, M]_{u}\right]
\end{aligned}
$$


Moving on to term (II) we find that

$$
\begin{aligned}
0 & =e^{-r t} S_{t}^{\alpha-1} \Lambda \Gamma B S_{t} U_{t}-\frac{\rho}{2} \mathbb{E}_{t}\left[\int_{t}^{T} e^{-r u} S_{t}^{\alpha-1} \Lambda \Gamma B S_{u} \sqrt{\nu_{u}} d[M, W]_{u}\right] \\
& +\frac{1}{2} \mathbb{E}_{t}\left[\int_{t}^{T} e^{-r u}\left(\frac{\Theta_{u}^{2}}{S^{2}}-\nu_{u}\right) \Gamma\left[S_{t}^{\alpha-1} \Lambda \Gamma B S_{u}\right] U_{u} d u\right] \\
& +\frac{\rho}{2} \mathbb{E}_{t}\left[\int_{t}^{T} e^{-r u} \Theta_{u} \Lambda \Gamma\left[S_{t}^{\alpha-1} \Lambda \Gamma B S_{u}\right] U_{u} d[M, W]_{u}\right] \\
& +\frac{1}{8} \mathbb{E}_{t}\left[\int_{t}^{T} e^{-r u} \Gamma^{2}\left[S_{t}^{\alpha-1} \Lambda \Gamma B S_{u}\right] U_{u} d[M, M]_{u}\right] \\
& +\frac{1}{2} \mathbb{E}_{t}\left[\int_{t}^{T} e^{-r u} \Theta_{u} \Lambda\left[S_{t}^{\alpha-1} \Lambda \Gamma B S_{u}\right] d[U, Z]_{u}\right] \\
& +\frac{1}{2} \mathbb{E}_{t}\left[\int_{t}^{T} e^{-r u} \Gamma\left[S_{t}^{\alpha-1} \Lambda \Gamma B S_{u}\right] d[U, M]_{u}\right]
\end{aligned}
$$

where $A_{t}=S_{t}^{\alpha-1} \Lambda \Gamma B S_{t}$ and $G_{t}=U_{t}=\frac{\rho}{2} \mathbb{E}_{t}\left[\int_{t}^{T} \sqrt{\nu_{u}} d[M, W]_{u}\right]$. Note that

$$
\begin{aligned}
U_{t} & =\frac{\rho \xi}{2 \kappa^{2}}\left(\theta \kappa(T-t)-2 \theta+\left(2 \theta-\nu_{t}\right) e^{-\kappa(T-t)}+\kappa(T-t)\left(\nu_{t}-\theta\right) e^{-\kappa(T-t)}\right) \\
d U_{t} & =\frac{\rho \xi^{2}}{2}\left(\int_{t}^{T} e^{-\kappa(z-t)} \varphi(z) d z\right) \sqrt{\nu_{t}} d W_{t}-\frac{\rho \xi}{2} \varphi(t)^{2} \nu_{t} d t .
\end{aligned}
$$

For term (III) set $A_{t}=\Gamma^{2} B S_{t}$ and $G_{t}=R_{t}=\frac{1}{8} \mathbb{E}_{t}\left[\int_{t}^{T} d[M, M]_{u}\right]$. Then

$$
\begin{aligned}
R_{t} & =\frac{\xi^{3}}{8 \kappa^{2}}\left(\theta(T-t)+\left(\nu_{t}-3 \theta\right) \varphi(t)+\left(\nu_{t}-\theta\right)(\varphi(t)-2) e^{-\kappa(T-t)}+\theta \varphi_{2}(t)\right) \\
d R_{t} & =\frac{\xi^{3}}{8} \sqrt{\nu_{t}}\left(\int_{t}^{T} e^{-\kappa(z-t)} \varphi(z)^{2} d z\right) d W_{t}-\frac{\xi^{2}}{8} \varphi(t)^{2} \nu_{t} d t
\end{aligned}
$$


Then we have,

$$
\begin{aligned}
0 & =e^{-r t} G_{t} \Gamma^{2} B S_{t}+\frac{1}{2} \mathbb{E}_{t}\left[\int_{t}^{T} e^{-r u}\left(\frac{\Theta_{u}^{2}}{S^{2}}-\nu_{u}\right) \Gamma\left[\Gamma^{2} B S_{u}\right] R_{u} d u\right] \\
& +\frac{\rho}{2} \mathbb{E}_{t}\left[\int_{t}^{T} e^{-r u} \Theta_{u} \Lambda \Gamma\left[\Gamma^{2} B S_{u}\right] R_{u} d[M, W]_{u}\right] \\
& +\frac{1}{8} \mathbb{E}_{t}\left[\int_{t}^{T} e^{-r u} \Gamma^{2}\left[\Gamma^{2} B S_{u}\right] R_{u} d[M, M]_{u}\right]-\frac{1}{8} \mathbb{E}_{t}\left[\int_{t}^{T} \Gamma^{2} B S_{u} d[M, M]_{u}\right] \\
& +\frac{1}{2} \mathbb{E}_{t}\left[\int_{t}^{T} e^{-r u} \Theta_{u} \Lambda\left[\Gamma^{2} B S_{u}\right] d[R, Z]_{u}\right]+\frac{1}{2} \mathbb{E}_{t}\left[\int_{t}^{T} e^{-r u} \Gamma\left[\Gamma^{2} B S_{u}\right] d[R, M]_{u}\right]
\end{aligned}
$$

From equations (.2), (.3), and (.4) above we find that the error term in proposition 2 is given as

$$
\begin{aligned}
\Omega & =\mathbb{E}_{t}\left[\int _ { t } ^ { T } e ^ { - r u } \left(f_{1} d u+f_{2} d[M, W]_{u}+f_{3} d[M, M]_{u}\right.\right. \\
& \left.\left.+f_{4} d[U, Z]_{u}+f_{5} d[U, M]_{u}+f_{6} d[R, Z]_{u}+f_{7} d[R, M]_{u}\right)\right]
\end{aligned}
$$

where

- $f_{1}=\frac{1}{4} C_{u}\left(\frac{\Theta_{u}^{2}}{S^{2}}-\nu_{u}\right) \Gamma\left(\left(S_{u}^{2(\alpha-1)}-1\right) \Gamma B S_{u}\right)+\frac{\xi}{4} \varphi(u) \sqrt{\nu_{u}} \Theta_{u} \Lambda\left(\left(S_{u}^{2(\alpha-1)}-\right.\right.$ 1) $\left.\Gamma B S_{u}\right)+\frac{1}{2}\left(\frac{\Theta_{u}^{2}}{S^{2}}-\nu_{u}\right) \Gamma\left[S_{t}^{\alpha-1} \Lambda \Gamma B S_{u}\right] U_{u}$

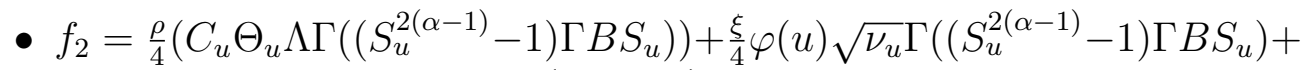
$\frac{\rho}{2} \Theta_{u} \Lambda \Gamma\left[S_{t}^{\alpha-1} \Lambda \Gamma B S_{u}\right] U_{u}+\frac{1}{2}\left(\frac{\Theta_{u}^{2}}{S^{2}}-\nu_{u}\right) \Gamma\left[\Gamma^{2} B S_{u}\right] R_{u}+\frac{\rho}{2} \Theta_{u} \Lambda \Gamma\left[\Gamma^{2} B S_{u}\right] R_{u}$,

- $f_{3}=\frac{1}{16} C_{u} \Gamma^{2}\left(\left(S_{u}^{2(\alpha-1)}-1\right) \Gamma B S_{u}\right)+\frac{1}{8} \Gamma^{2}\left[S_{t}^{\alpha-1} \Lambda \Gamma B S_{u}\right] U_{u}+\frac{1}{8} \Gamma^{2}\left[\Gamma^{2} B S_{u}\right] R_{u}$,

- $f_{4}=\frac{1}{2} \Theta_{u} \Lambda\left[S_{t}^{\alpha-1} \Lambda \Gamma B S_{u}\right], \quad f_{5}=\frac{1}{2} \Gamma\left[S_{t}^{\alpha-1} \Lambda \Gamma B S_{u}\right]$,

- $f_{6}=\frac{1}{2} \Theta_{u} \Lambda\left[\Gamma^{2} B S_{u}\right]$, and $f_{7}=\frac{1}{2} \Gamma\left[\Gamma^{2} B S_{u}\right] d[R, M]_{u}$.

\section{References}

F. Black, M. Scholes, The pricing of options and corporate liabilities, Journal of Political Economy 81 (1973) 637-654. 
J. C. Cox, Notes on option pricing i: Constant elasticity of diffusions, 1975. Unpublished Draft, Stanford University.

S. L. Heston, A closed-form solution for options with stochastic volatility with applications to bond and currency options, Review of Financial Studies (1993).

E. Alòs, A decomposition formula for option prices in the heston model and applications to option pricing approximation, Finance and Stochastics 16 (2012) 403-422.

R. Merino, J. Vives, A generic decomposition formula for pricing vanilla options under stochastic volatility models, International Journal of Stochastic Analysis 2015 (2015).

R. Merino, J. Vives, Option price decomposition in spot-dependent volatility models and some applications, International Journal of Stochastic Analysis 2017 (2017).

S.-Y. Choi, J.-P. Fouque, J.-H. Kim, Option pricing under hybrid stochastic and local volatility, Quantitative Finance 13 (2013) 1157-1165.

Y. El-Khatib, A. Hatemi-J, An alternative stochastic volatility model, in: European Consortium for Mathematics in Industry, Springer, 2014, pp. 1029-1036.

R. Lord, R. Koekkoek, D. V. Dijk, A comparison of biased simulation schemes for stochastic volatility models, Quantitative Finance 10 (2010) 177-194.

M. Broadie, Ö. Kaya, Exact simulation of stochastic volatility and other affine jump diffusion processes, Operations research 54 (2006) 217-231.

L. B. Andersen, Efficient simulation of the heston stochastic volatility model (2007).

A. Medvedev, O. Scaillet, Pricing american options under stochastic volatility and stochastic interest rates, Journal of Financial Economics 98 (2010) $145-159$. 